\title{
Individual and household factors associated with incidences of village malaria in Xepon district, Savannakhet province, Lao PDR
}

Nouhak Inthavong 1,2,3, Daisuke Nonaka ${ }^{1,2^{*}}$, Sengchanh Kounnavong ${ }^{3}$, Moritoshi Iwagami ${ }^{2,4,5}$, Souraxay Phommala ${ }^{3}$, Jun Kobayashi, ${ }^{1,2}$, Bouasy Hongvanthong ${ }^{2,6}$, Tiengkham Pongvongsa ${ }^{2,7}$, Paul T. Brey ${ }^{2,5}$ and Shigeyuki Kano ${ }^{2,4}$

\begin{abstract}
Background: In the Lao PDR, the incidence of malaria greatly differs among villages even within a subdistrict, and the reasons for this difference are poorly understood. The objective of this study was to identify differences in villagers' behavior and the household environment between villages with high incidences and those with low incidences of malaria in a rural district of the Lao PDR.

Methods: A case-control study was conducted in Xepon district, Savannakhet province. Case villages were defined as those with a high incidence (> 10 cases per 1000 population per year), and control villages were those with a low incidence (0-10 cases per 1000 population per year). Data were collected from 178 households in the six case villages and six control villages between December 2016 and January 2017. The data collection consisted of an interview survey with the heads of households and an observational survey in and around the house. Logistic regression was used to assess the association between the case-control status and individual-level behavioral factors and household-level environmental factors adjusted for socio-demographic and economic factors.

Results: Compared to the household members in the control villages, household members in the case villages were significantly more likely to work at night in the forest (adjusted odds ratio 1.95; 95\% confidence interval 1.28 to 2.98) and more likely to sleep overnight in the forest (adjusted odds ratio 1.94; 95\% confidence interval 1.13 to 3.33). Additionally, compared to the households in the control villages, households in the case villages were significantly more likely to have an open space on the house surface (adjusted odds ratio 3.64; $95 \%$ confidence interval 1.68 to 7.84 ).

Conclusions: There were significant differences in nighttime working and sleeping behaviors in the forest and the presence of an open space on the house surface in the case versus control villages. These differences can partly explain the difference in the incidences of malaria among the villages. The Lao National Malaria Control Program should recommend that villagers use personal protection when working and sleeping in the forest and to reduce any open space on the house surfaces.
\end{abstract}

Keywords: Malaria, Incidence, Risk factor, Behavior and Laos

\footnotetext{
* Correspondence: laodaisuke@hotmail.co.jp

'Department of Global Health, Graduate School of Health Sciences,

University of the Ryukyus, Uehara 207, Nishihara-cho, Okinawa 903-0215,

Japan

${ }^{2}$ SATREPS Project for Parasitic Diseases, Vientiane, Lao People's Democratic

Republic

Full list of author information is available at the end of the article
} 


\section{Background}

The Lao People's Democratic Republic (Lao PDR) is a lower-middle-income country in Southeast Asia, bordering with China on its north border, Vietnam in the east and northeast, Cambodia in the south, Thailand in the west, and Myanmar in the northwest (Fig. 1). The country comprises 18 provinces with 148 districts and 8500 villages. The total land area is 23.68 million hectares, $79 \%$ of which is mountainous. The population of the Lao PDR was 7.0 million in 2016 [1], of which $80 \%$ live in rural areas and $85-90 \%$ are dependent upon subsistence farming [2].

Since 1992, the Lao PDR has implemented a nationwide malaria control program. The current malaria control strategies emphasize the promotion of long-lasting insecticide-treated bed nets (LLINs), early diagnosis by microscopic examination and rapid diagnostic tests, and prompt treatment with an artemisinin-based combination therapy (ACT). Since 2008, the use of the ACT has gradually been scaled-up to cover the entire public health sector, including village health volunteers (community health workers), some businesses in the private sector, and registered private pharmacies [3].

Since 2010, the Lao National Malaria Control Program has adopted stratification-based planning and implementation of control activities; villages are stratified into three strata according to village-level incidences of malaria, and different control strategies are applied to different strata. In stratum I villages, which are defined by an annual incidence of less than 0.1 cases per 1000 population, control activities focus on the maintenance of existing bed nets. In stratum II villages, which are defined by an annual incidence of 0.1 to 10 cases per 1000 population, control activities include the distribution of LLINs and the implementation of village-level diagnosis and treatment with rapid diagnostic tests and ACTs, respectively, and the maintenance of existing bed nets. In stratum III villages, which are defined by an annual incidence of more than 10 cases per 1000 population, additional control activities are the distribution of single LLINs to mobile members of the population and the provision of insect repellent [3].

During the period between 2000 and 2010, the Lao PDR significantly reduced its malaria burden by reducing the number of annual malaria deaths from 350 to 24. The number of confirmed cases had also declined from around 75,000 cases in 2000 to 30,000 cases in 2010. However, the national annual parasite incidence rose from 2.66/1000 population in 2011 to 7.3/1000 population in 2014 [4]. Currently, the malaria burden is concentrated in the southern provinces; approximately $90 \%$ of the malaria cases were reported in the five southernmost provinces, i.e., Savannakhet, Saravan, Sekong, Attapeu, and Champasak [3, 5].

In a total of 15 districts in Savannakhet province, malaria was endemic in four districts including Xepon district, which is located on the Vietnamese border, approximately $600 \mathrm{~km}$ to the southeast of the Vientiane capital. Xepon has a total area of $21,774 \mathrm{~km}^{2}$ and 45,000 people. According to the Xepon District Health Office, most of them are ethnic minorities, specifically the Tri and Mangkong people, comprising $75 \%$ of the total district population. They have their own distinctive languages, have limited formal education, and live in mountainous and forested areas that are far from health care facilities. The majority of the population is farmers

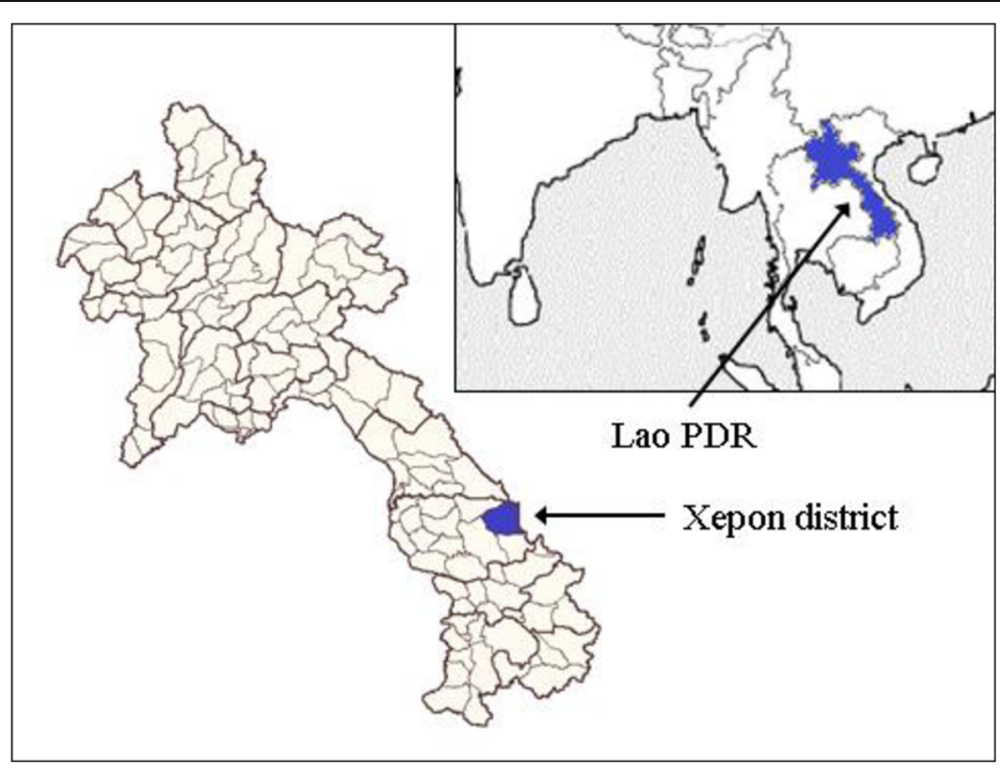

Fig. 1 The location of the Lao PDR and Xepon district 
engaged in rice farming. Their houses are surrounded by vegetation and puddles, and cattle are often kept in fenced enclosures near the houses. According to the surveillance data obtained from the Center of Malariology, Parasitology and Entomology, the Xepon District Hospital recorded 225 cases of malaria including 208 Plasmodium falciparum mono-infections, 16 Plasmodium vivax mono-infections, and 6 co-infections of $P$. falciparum and P. vivax in 2015.

A number of studies conducted in the Lao PDR or neighboring countries have emphasized the importance of behavioral and environmental factors on the risk of malaria infection. In the Lao PDR, inappropriate use of bed nets [6] and sleeping away from home (e.g., sleeping in a farming hut) have been reported as risk factors [7, 8]. In Thailand, the relative risk of malaria infection was three times higher among people who slept in farm huts than in people staying in residential villages [9]. In Sri Lanka, the incidence of malaria among residents of poor-quality housing was higher compared with a population living in improved housing [10]. A study conducted in the Lao PDR also showed that house construction material, veranda style, kitchen location, and cow ownership were significantly associated with the house entry of Anopheles mosquitoes [11]. In addition, a study in Vietnam mentioned that living in a wooden/bamboo house was significantly related to malaria infection [12]. Hence, the present study hypothesized that individual behavioral factors and household environmental factors would be significantly different between villages with high incidences and villages with low incidences of malaria in Xepon district, Savannakhet province, Lao PDR.

\section{Methods}

\section{Study site and population}

This was a case-control study that was conducted in the 12 villages in the catchment area of the Dongsavanh Health Center, Xepon district (Fig. 2). A case village was defined as a stratum III village, and the control villages were defined as stratum I or II villages. Of the 43 villages in the catchment area, 13 stratum I and II villages were excluded, either because the number of households was less than the required number for a survey or because these villages were located near the health center and were thus not comparable to stratum III villages that were located far from the health center. Then, 6 case villages were randomly selected from 19 stratum III villages. Similarly, 6 control villages were randomly selected from 11 stratum I and II villages, 3 from the 7 stratum I villages and 3 from the 4 stratum II villages. The number of villages and households to be selected was mainly determined by the availability of human and time resources.

In each of the selected villages, 15 households were randomly selected, using a household list obtained from the heads of the villages; however, the survey failed to include 15 households in one village. All members of the selected households were included. Overall, this study collected data on 1070 individuals from 178 households.

The names (and number of eligible households) of the selected case villages were Lakheum-Tai (26), Palai (52), Alang-Noy (22), Satheum (53), Ahor (39), and Kaengkhai (43), and those of the selected control villages were Haengluoang (17), Kaengpae-Yai (18), Sky-Yai (60), Vangsalor (20), Tanang (33), and Alai-Noy (34).

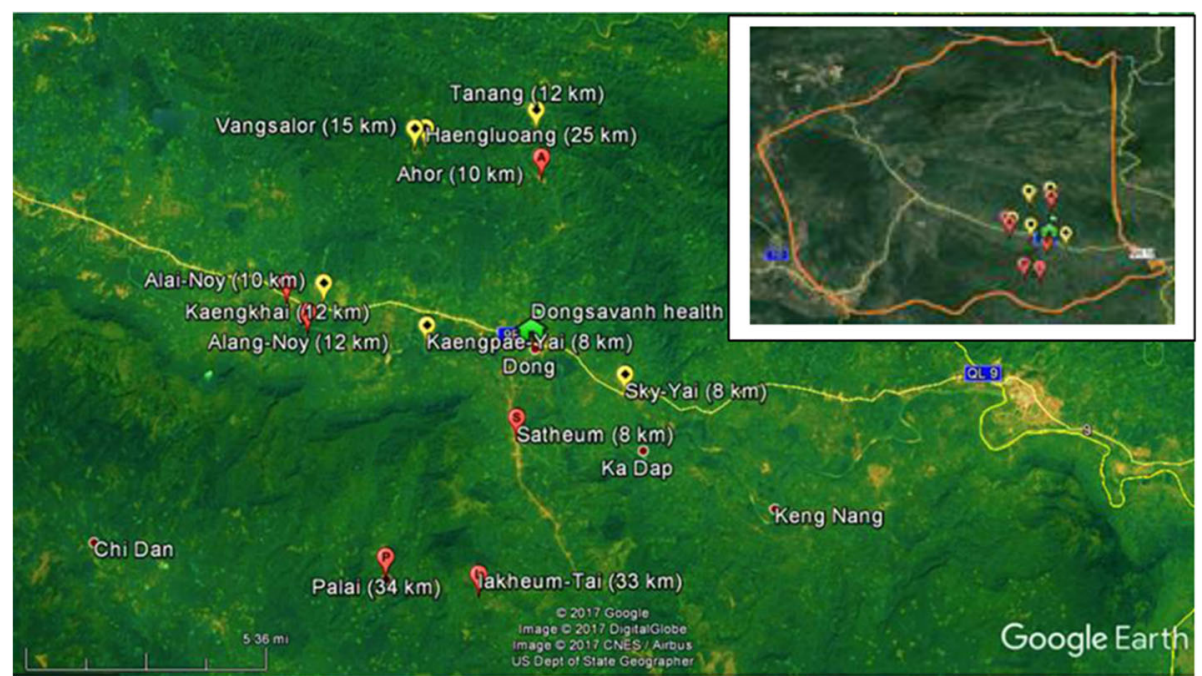

Fig. 2 Map of the study villages. The maps show the villages in which the present study was conducted. The red points indicate the locations of case villages, and the yellow points indicate the locations of control villages along with the distance of each from Xepon district hospital according to the hospital's data 


\section{Data collection}

Data were collected between December 2016 and January 2017 through an interview survey and an observational survey. The trained surveyors, who were health workers at the Xepon District Health Office, visited the selected households and conducted interviews with the heads of households. Information on every member of each household was collected via the heads of households. When the head of a household was absent during the survey, another adult member of the household (e.g., spouse of the head) was recruited. When the respondent was unable to communicate with surveyors in the Lao language, the head of the village or other villagers who were able to speak both the local language and the Lao language were asked to assist the surveyors. For the observational survey, surveyors observed the household characteristics in and around the houses using a checklist.

\section{Factors and measurements}

Selection of the factors that were measured in this study was based on the conceptual framework depicted in Fig. 3. The conceptual model was developed principally on the basis of the findings of malaria studies that were conducted in the Lao PDR or neighboring countries [614]. The interview survey measured socio-demographic and economic factors and individual behavioral factors, whereas the observational survey measured household environmental factors.

Individual behavioral factors included preventive behavior (i.e., frequency of sleeping under a bed net in the past month), occupational behavior (i.e., experience of working in the forest at night or early morning in the past 1 year, sleeping in the forest in the past 1 year), and seeking behavior for malaria treatment in the past 1 year.

Household environmental factors included housing structure (i.e., wall and floor materials); presence of an open space that was larger than the size of an A4 sheet of paper on the surface of the house (i.e., lack of a door or no window coverings, no ceiling, and open eaves); kitchen location; type of water resources for bathing, washing clothes, and other daily use; keeping cattle near the house; presence of weeds within $10 \mathrm{~m}$ of the house in the household compound; and household bed net density (i.e., person per net ratio defined as the number of household members divided by the number of available bed nets in the household [15]). When measuring the presence of an open space, surveyors paid attention to and included only an open space that was larger than approximately the size of an A4 sheet of paper, ignoring an open space that was smaller than approximately the size of an A4 sheet of paper.

Socio-demographic factors included age, gender, and number of members in each household. Socioeconomic factors included educational attainment, occupation of each member of the households, and household relative wealth determined by the household possession of assets including a motorbike, mobile phone, television, DVD/CD, car/tractor, and refrigerator. Principal component analysis was used to assess the weight of household assets by scoring factors for wealth index variables (Table 1) and to build a household wealth index [16]. Households were ranked by the household wealth index and divided into quartiles. As a result of the analysis, six components were extracted and the first component, which explained $41.3 \%$ of the total variance with the eigenvalue of 2.48, was used for the wealth index. For each asset variable, a scoring factor was derived from the weight of a variable divided by the eigenvalue. The wealth index of a household was expressed as the sum of the product between the scoring factor of an

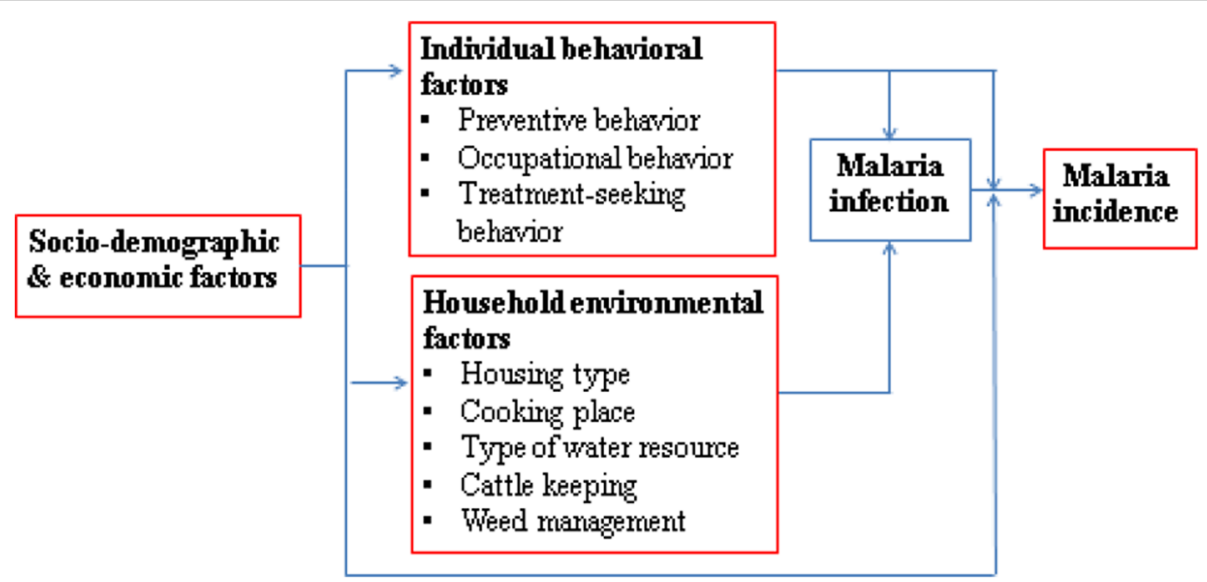

Fig. 3 Conceptual framework 
Table 1 Scoring factors for wealth index variables

\begin{tabular}{ll}
\hline Variables & Scoring factor \\
\hline Car/tractor & 0.216 \\
Motorbike & 0.232 \\
Mobile phone & 0.262 \\
Television & 0.323 \\
DVD/CD & 0.301 \\
Refrigerator & 0.198 \\
\hline
\end{tabular}

asset variable and the household's standardized score of the variable.

\section{Data analysis}

Frequencies, percentages, and medians with interquartile ranges were computed to summarize the data. Bivariate analyses were conducted to assess an association between a predictive factor and the case-control status, using Fisher's exact test. Multivariate analyses that adjusted for the effect of other predictive factors were conducted to assess an association between a predictive factor and the case-control status using logistic regression. According to Katz [17], regardless of a $p$ value obtained from the bivariate analyses, variables that are theoretically important or those that have been reported as risk factors were included in the multivariate model. Two multivariate models were built: one included individual-level predictive variables and the other included only household-level predictive variables. The amount of multicollinearity (i.e., two or more predictive variables are correlated each other) in the multivariate model was estimated by the variance inflation factor (VIF). A VIF $\geq 4$ was considered as the sign of multicollinearity. A $p$ value $<0.05$ was considered statistically significant. Statistical analyses were performed using SPSS version 24 .

\section{Results}

Associations between individual-level characteristics and case-control status

The proportion of household members who worked in the forest at night was significantly higher in the case villages than in the control villages (21.8 vs. $15.6 \%$, $p=0.010$ ) (Table 2). In contrast, the proportion of household members who worked in the forest in the early morning was significantly lower in the case villages than in the control villages (19.1 vs. $24.4 \%, p=0.038)$. Additionally, the proportion of household members who slept overnight in the forest $(12.4$ vs. $8.1 \%, p=0.026)$, who had a malaria episode in past 1 year ( 13.8 vs. $6.3 \%$, $p=<0.001)$, and who sought a place for malaria treatment (48.7 vs. $0.0 \%, p=<0.001)$ was significantly higher in the case villages versus the control villages. No significant differences were observed in gender, age,
Table 2 Bivariate analysis of differences in individual-level characteristics

\begin{tabular}{|c|c|c|c|c|c|}
\hline \multirow[t]{2}{*}{ Characteristics } & \multicolumn{2}{|c|}{$\begin{array}{l}\text { Respondents in } \\
\text { cases }(n=550)\end{array}$} & \multicolumn{2}{|c|}{$\begin{array}{l}\text { Respondents in } \\
\text { controls } \\
(n=520) \\
\end{array}$} & \multirow[t]{2}{*}{$\begin{array}{l}p \\
\text { value }^{a}\end{array}$} \\
\hline & $n$ & $\%$ & $n$ & $\%$ & \\
\hline \multicolumn{6}{|l|}{ Gender } \\
\hline Male & 272 & 49.5 & 261 & 50.2 & \multirow[t]{2}{*}{0.854} \\
\hline Female & 278 & 50.5 & 259 & 49.8 & \\
\hline \multicolumn{6}{|l|}{ Age } \\
\hline$<15$ years & 271 & 50.4 & 239 & 47.0 & \multirow[t]{2}{*}{0.293} \\
\hline$\geq 15$ years & 267 & 49.6 & 270 & 53.0 & \\
\hline \multicolumn{6}{|l|}{ Educational attainment } \\
\hline No formal education & 389 & 70.7 & 367 & 70.6 & \multirow[t]{2}{*}{1.000} \\
\hline Primary school or higher & 161 & 29.3 & 153 & 29.4 & \\
\hline \multicolumn{6}{|l|}{ Occupation } \\
\hline Farmer & 290 & 52.7 & 275 & 52.9 & \multirow[t]{3}{*}{0.978} \\
\hline Child & 159 & 28.9 & 152 & 29.2 & \\
\hline Student & 101 & 18.4 & 93 & 17.9 & \\
\hline \multicolumn{6}{|l|}{ Frequency of using a bed net } \\
\hline Everyday & 455 & 82.7 & 428 & 82.3 & \multirow[t]{2}{*}{0.872} \\
\hline Sometimes/never & 95 & 17.3 & 92 & 17.7 & \\
\hline \multicolumn{6}{|c|}{ Working in the forest at nighttime in the past year } \\
\hline No & 430 & 78.2 & 439 & 84.4 & \multirow[t]{2}{*}{0.010} \\
\hline Yes & 120 & 21.8 & 81 & 15.6 & \\
\hline
\end{tabular}

Working in the forest in the early morning in the past year

$\begin{array}{llllll}\text { No } & 445 & 80.9 & 393 & 75.6 & 0.038\end{array}$

$\begin{array}{lllll}\text { Yes } & 105 & 19.1 & 127 & 24.4\end{array}$

Sleeping overnight in the forest in the past year

$\begin{array}{lccccc}\text { No } & 482 & 87.6 & 478 & 91.9 & 0.026 \\ \text { Yes } & 68 & 12.4 & 42 & 8.1 & \\ \text { Malaria episode in past 1 year } & & & & \\ \text { No } & 474 & 86.2 & 487 & 93.7 & <0.001 \\ \text { Yes } & 76 & 13.8 & 33 & 6.3 & \end{array}$

Place for the treatment of the episode

\begin{tabular}{llllll} 
Health center & 36 & 47.4 & 30 & 90.9 & $<0.001$ \\
Village health volunteer & 37 & 48.7 & 0 & 0.0 & \\
Other & 3 & 3.9 & 3 & 9.1 & \\
\hline
\end{tabular}

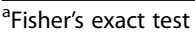

educational attainment, occupation, and frequency of using a bed net.

Among the household members who worked in the forest at night, the proportion of household members who slept overnight in the forest was not significantly different between the case and control villages (43.3 vs. $48.1 \%, p=0.564$ ) (data not shown in table).

After adjusting for the effects of other individual-level predictive variables in the multivariate analysis, the 
associations observed in the bivariate analysis remained significant (Table 3); working in the forest at night (adjusted odds ratio [AOR] 1.95; 95\% confidence interval [CI] 1.28 to 2.98), working in the forest in the early morning (AOR 0.51; 95\% CI 0.36 to 0.73), and sleeping overnight in the forest (AOR 1.94; 95\% CI 1.13 to 3.33). Although the difference in age groups was not significant in the bivariate analysis, it became significant in the multivariate analysis (AOR 0.61; 95\% CI 0.38 to 0.98). There was no sign of multicollinearity between the variables in the multivariate analysis.

\section{Associations between household-level characteristics and case-control status}

The proportion of households that used flooring materials other than wood was significantly lower in the case villages than in the control villages (21.1 vs. $36.4 \%$,

Table 3 Multivariate analysis of differences in individual-level characteristics and case-control status

\begin{tabular}{|c|c|c|}
\hline Predictive factors & $\begin{array}{l}\text { Crude odds ratio } \\
(95 \% \mathrm{Cl})\end{array}$ & $\begin{array}{l}\text { Adjusted odds ratio } \\
(95 \% \mathrm{Cl})\end{array}$ \\
\hline \multicolumn{3}{|l|}{ Gender } \\
\hline Male & 1.00 & 1.00 \\
\hline Female & $0.97(0.76-1.23)$ & $1.22(0.93-1.61)$ \\
\hline \multicolumn{3}{|l|}{ Age } \\
\hline$<15$ years & 1.00 & 1.00 \\
\hline$\geq 15$ years & $0.87(0.68-1.11)$ & $0.61(0.38-0.98)$ \\
\hline \multicolumn{3}{|l|}{ Education attainment } \\
\hline No formal education & 1.00 & 1.00 \\
\hline Primary school and higher & $0.99(0.76-1.29)$ & $0.88(0.58-1.32)$ \\
\hline \multicolumn{3}{|l|}{ Occupation } \\
\hline Child & 1.00 & 1.00 \\
\hline Student & $1.03(0.72-1.48)$ & $1.33(0.78-2.25)$ \\
\hline Farmer & $1.00(0.76-1.33)$ & $1.46(0.87-2.45)$ \\
\hline \multicolumn{3}{|l|}{ Frequency of using bed net } \\
\hline Everyday & 1.00 & 1.00 \\
\hline Sometimes/never & $0.97(0.70-1.33)$ & $0.80(0.57-1.14)$ \\
\hline \multicolumn{3}{|c|}{ Working in the forest at nighttime in the past year } \\
\hline No & 1.00 & 1.00 \\
\hline Yes & $1.51(1.10-2.06)$ & $1.95(1.28-2.98)$ \\
\hline \multicolumn{3}{|c|}{ Working in the forest in the early morning in the past year } \\
\hline No & 1.00 & 1.00 \\
\hline Yes & $0.73(0.54-0.97)$ & $0.51(0.36-0.73)$ \\
\hline \multicolumn{3}{|c|}{ Sleeping overnight in the forest in the past year } \\
\hline No & 1.00 & 1.00 \\
\hline Yes & $1.60(1.07-2.40)$ & $1.94(1.13-3.33)$ \\
\hline
\end{tabular}

$p=0.031)$. The proportion of households with an open space was significantly higher in the case villages than in the control villages ( 77.8 vs. $50.0 \%, p=<0.001$ ) (Table 4 ). No other significant differences were found in the bivariate analysis.

After adjusting for the effects of other household-level variables in the multivariate analysis, the association with flooring materials other than wood was significant (AOR 0.25; 95\% CI 0.09-0.68) (Table 5). The presence of an open space also remained significant (AOR 3.64; 95\% CI 1.68 to 7.84 ). Although the bivariate analysis did not show a significant difference in the proportion of household wealth quartiles, the multivariate analysis showed that compared to the proportion of the firstquartile households, the proportion of the fourthquartile households was significantly higher in the case villages than in the control villages (AOR 6.16; 95\% CI 1.92 to 19.72). There was no sign of multicollinearity between the variables in the multivariate analysis.

\section{Risk behaviors in households}

There was no tendency that risk behaviors concentrated in some particular households; $68.9 \%(62 / 90)$ of the households in the case villages and $67.0 \%(59 / 88)$ of the households in the control villages had at least one member who worked in the forest at night. Additionally, $51.1 \%(46 / 90)$ of the households in the case villages and $37.5 \%(33 / 88)$ of the households in the control villages had at least one member who slept in the forest at night (data not shown in table).

\section{Discussion}

This study found that a number of individual-level and household-level factors were associated with village-level incidences of malaria. The results showed that the household members in the case villages were significantly more likely to work at night and more likely to sleep overnight in the forest, as compared with those in the control villages. These differences of behaviors could be the reason why the incidences of malaria were higher in the case villages than in the control villages. A casecontrol study conducted in Attapeu province, Lao PDR reported that study participants who slept away from home in the past 2 weeks had significantly higher odds of malaria infection than those who did not $[7,8]$. A cross-sectional study that was conducted in Savannakhet province, Lao PDR reported that staying overnight in the forest was associated with $P$. viviax mono-infections [18]. A cross-sectional study conducted in Vietnam reported that working and sleeping at night in the forest was a risk factor for malaria infection [13], and a similar finding was also reported from Thailand [14]. In the study district, Anopheles dirus is thought to be the main malaria vector [19]. The biting time of $A$. dirus is 
Table 4 Bivariate analysis of the associations between household-level characteristics

\begin{tabular}{|c|c|c|c|}
\hline \multirow[t]{2}{*}{ Characteristics } & $\begin{array}{l}\text { Respondents } \\
\text { in cases } \\
(n=90)\end{array}$ & $\begin{array}{l}\text { Respondents } \\
\text { in controls } \\
(n=88)\end{array}$ & $p$ value $^{a}$ \\
\hline & $\%$ & $\%$ & \\
\hline
\end{tabular}

\begin{tabular}{|c|c|c|c|c|c|}
\hline \multicolumn{6}{|l|}{ Number of household members } \\
\hline$<5$ & 27 & 30.0 & 27 & 30.7 & 0.994 \\
\hline $5-7$ & 40 & 44.4 & 39 & 4.3 & \\
\hline$\geq 8$ & 23 & 25.6 & 22 & 25.0 & \\
\hline Median (interquartile range) & 6 & $4-8$ & 6 & $4-8$ & \\
\hline \multicolumn{6}{|l|}{ Household that hung a bed net } \\
\hline No & 0 & 0.0 & 4 & 4.5 & 0.058 \\
\hline Yes & 90 & 100 & 84 & 95.5 & \\
\hline
\end{tabular}

Household that hung long-lasting insecticide-treated bed net

$\begin{array}{lllll}\text { No } & 3 & 3.3 & 9 & 10.2 \\ \text { Yes } & 87 & 96.7 & 79 & 89.8\end{array}$

0.079

Person per net ratio

$\begin{array}{lllll}\leq 2.5 & 49 & 54.4 & 44 & 52.4 \\ >2.5 & 41 & 45.6 & 40 & 47.6\end{array}$

Type of water used for daily life

$\begin{array}{lllll}\text { Stream water } & 41 & 45.6 & 44 & 50.0 \\ \text { Water other than stream } & 49 & 54.4 & 44 & 50.0\end{array}$

water

Wealth index quartiles

$\begin{array}{llllll}\text { First (least poor) } & 19 & 21.1 & 30 & 34.1 & 0.121 \\ \text { Second } & 21 & 23.3 & 22 & 25.0 & \\ \text { Third } & 18 & 20.0 & 17 & 19.3 & \\ \text { Fourth (poorest) } & 32 & 35.6 & 19 & 21.6 & \\ \begin{array}{l}\text { Wall materials } \\ \text { Brick }\end{array} & 48 & 53.3 & 45 & 51.1 & 0.881 \\ \text { Other } & 42 & 46.7 & 43 & 48.9 & \\ \text { Fooring materials } & & & & & \\ \text { Wood } & 71 & 78.8 & 56 & 63.6 & 0.031 \\ \text { Other } & 19 & 21.1 & 32 & 36.4 & \\ \text { Roofing materials } & & & & & \\ \text { Zinc } & 82 & 91.1 & 73 & 83.0 & 0.121 \\ \text { Other } & 8 & 8.9 & 15 & 17.0 & \end{array}$

Open space in the house (size larger than A4 sheet)

$\begin{array}{lllll}\text { No } & 20 & 22.2 & 44 & 50.0 \\ \text { Yes } & 70 & 77.8 & 44 & 50.0\end{array}$

Percent of open spaces in the house (\%)

$\begin{array}{llllll}\leq 10 & 23 & 32.9 & 13 & 29.5 & 0.837 \\ >10 & 47 & 67.1 & 31 & 70.5 & \\ \begin{array}{l}\text { Median (interquartile } \\ \text { range) }\end{array} & 11.2 & 0.9-21.5 & 0.6 & 0.0-18.4 & \end{array}$

Table 4 Bivariate analysis of the associations between household-level characteristics (Continued)

\begin{tabular}{|c|c|c|c|c|c|}
\hline \multirow[t]{2}{*}{ Characteristics } & \multicolumn{2}{|c|}{$\begin{array}{l}\text { Respondents } \\
\text { in cases } \\
(n=90)\end{array}$} & \multicolumn{2}{|c|}{$\begin{array}{l}\text { Respondents } \\
\text { in controls } \\
(n=88)\end{array}$} & \multirow[t]{2}{*}{$p$ value $^{a}$} \\
\hline & $n$ & $\%$ & $n$ & $\%$ & \\
\hline \multicolumn{6}{|l|}{ Kitchen location } \\
\hline Inside the house & 58 & 64.4 & 61 & 69.3 & \multirow[t]{2}{*}{0.527} \\
\hline Outside the house & 32 & 35.6 & 27 & 30.7 & \\
\hline \multicolumn{6}{|c|}{ Cattle keeping near the house } \\
\hline No & 53 & 58.9 & 58 & 65.9 & \multirow[t]{2}{*}{0.357} \\
\hline Yes & 37 & 41.1 & 30 & 34.1 & \\
\hline \multicolumn{6}{|c|}{ Weed management within $10 \mathrm{~m}$ around the house } \\
\hline Poor & 52 & 57.8 & 42 & 47.7 & \multirow[t]{2}{*}{0.230} \\
\hline Good & 38 & 42.2 & 46 & 52.3 & \\
\hline
\end{tabular}

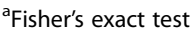

between 19:00 and 06:00 with the peak at midnight $[8,20]$. This vector behavior can also support our causal inference that the statistical associations with working and sleeping at night in the forest represent a cause-effect relation.

In rural areas of the Lao PDR, people work in the forest during nighttime to sustain their lives. The purposes of nighttime working in the forest include seeking forest products, hunting, and protecting crops in a slash-andburn field from animals. Therefore, it is difficult to stop their behavior. Considering the risk of malaria infection of the behavior, the Lao National Malaria Control Program has already promoted devices for protection. Mosquito repellent lotions and single LLINs for this mobile population have been made available in the stratum III villages where the incidences of malaria are high. However, little is known about whether the villagers are actually using these devices. In addition to these protective devices, wearing long-sleeved shirts should be recommended to villagers who work in the forest at nighttime. A cross-sectional study that was conducted with hill tribes in Thailand showed that when working outdoors, people who wore long-sleeved clothes were significantly less likely to have malaria infection, as compared with those who did not [21].

The present study also showed that the houses in the case villages were significantly more likely to have an unprotected open space on their surfaces, as compared to the houses in the control villages. The difference in the presence of an open space between the case and control houses could be one reason for the higher incidence of malaria in the case villages than in the control villages. Such open spaces can make it easier for vector mosquitoes to enter the house, thus increasing humanvector contacts. Although no study has been conducted in the Lao PDR and neighboring countries, a number of 
Table 5 Multivariate analysis of the associations between household characteristics and case-control status

\begin{tabular}{lll}
\hline Predictive factors & Crude odds & $\begin{array}{l}\text { Adjusted odds } \\
\text { ratio } \\
\text { ratio } \\
(95 \% \mathrm{Cl})\end{array}$ \\
& $(95 \% \mathrm{Cl})$ \\
\hline
\end{tabular}

Number of household members

$\begin{array}{lll}\leq 6 & 1.00 & 1.00 \\ >6 & 1.16(0.63-2.12) & 1.48(0.70-3.13) \\ \text { Person per net ratio } & & \\ \leq 2.5 & 1.00 & 1.00 \\ >2.5 & 0.92(0.50-1.67) & 0.83(0.40-1.71)\end{array}$

Type of water used for daily life

Stream water

$1.00 \quad 1.00$

Water other than stream water

$1.19(0.66-2.15) \quad 1.50(0.70-3.21)$

Wealth index quartiles

$\begin{array}{lll}\text { First (least poor) } & 1.00 & 1.00 \\ \text { Second } & 1.50(0.65-3.45) & 1.67(0.63-4.38) \\ \text { Third } & 1.67(0.69-4.02) & 2.35(0.77-7.14) \\ \text { Fourth (poorest) } & 2.65(1.18-5.96) & 6.16(1.92-19.72) \\ \text { Wall materials } & & \\ \text { Brick } & 1.00 & 1.00 \\ \text { Other } & 0.96(0.50-1.64) & 0.97(0.40-2.35) \\ \text { Flooring materials } & & \\ \text { Wood } & 1.00 & 1.00 \\ \text { Other } & 0.46(0.24-0.91) & 0.25(0.09-0.68) \\ \text { Roofing materials } & & \\ \text { Zinc } & 1.00 & 1.00 \\ \text { Other } & 0.47(0.19-1.18) & 0.61(0.21-1.75)\end{array}$

Open space in the house

$\begin{array}{lll}\text { No } & 1.00 & 1.00 \\ \text { Yes } & 3.50(1.82-6.70) & 3.64(1.68-7.84)\end{array}$

Kitchen location

Inside the house

$1.00 \quad 1.00$

Outside the house

$1.24(0.66-2.33) \quad 0.94(0.35-2.48)$

Cattle keeping near the house

No

Yes

$1.00 \quad 1.00$

$1.35(0.73-2.48) \quad 1.78(0.85-3.74)$

Weed management within $10 \mathrm{~m}$ around the house

Poor

1.00

1.00

Good

$0.66(0.36-1.20)$

$0.67(0.32-1.39)$

${ }^{a}$ Adjusted for number of household members, person per net ratio, type of water used for daily life, wealth index, wall materials, flooring materials, roofing materials, open space in the house, kitchen location, cattle keeping near the house, weed management within $10 \mathrm{~m}$ around the house

studies conducted in Sri Lanka or African countries have shown an association between the presence of an open space or non-screened space on the surface of a house and malaria infections among the residents of the house
$[10,22-25]$. An open space under the eaves is considered to be the most critical for house entry of malaria vectors $[23,25,26]$. A randomized controlled trial of house screening intervention showed that the number of mosquitoes inside the house and the incidences of childhood anemia were significantly lowered in the intervention group [23].

There are at least two concerns about promoting net screens in the study district. One is the increased temperature due to installing net screens. A study in Gambia showed that houses with net screens were a little hotter than houses without any screens; full screened houses were $0.26{ }^{\circ} \mathrm{C}$ hotter at night than houses with screened ceilings and $0.51{ }^{\circ} \mathrm{C}$ hotter than houses with no screening $\left(28.43{ }^{\circ} \mathrm{C}\right)$. Nonetheless, only $9 \%$ of full screened house users complained about the heat [27]. Another concern is the cost for installing net screens. Because many households in malaria-prone villages of the study district may not afford to buy nets for screening by themselves, the Lao National Malaria Control Program should consider subsidizing a part of the cost. The Lao National Malaria Control Program has already experienced the distribution of bed nets and repellent spays to the target population at a subsidized price.

Although the difference in household bed net density measured by person per net ratio was not significant between the case and controls, it should be noted that $45.6 \%$ of the households in the case villages did not possess an adequate number of bed nets to protect all members of the household. This finding suggests the need for increasing the availability of LLINs in malariaprone villages.

In the present study, the poorest households were significantly more abundant in the case villages than in the control villages. It has been frequently reported from many countries that poorer households are at higher risk of malaria [26, 28, 29]. In this study site, there are at least two possible explanations for why poor households are at risk of malaria. First, poor households would be more reluctant to seek treatment for malaria as compared to non-poor households. Although treatment for malaria is free of charge in the Lao PDR, treatment seeking requires opportunity costs including transportation costs. Second, as compared to better-off households, poor households would be more likely to be exposed to malaria infection because protective materials including long-sleeved clothes or insecticides are less likely available among these households.

The present study compared individual and household characteristics between the case villages and control villages. The result showed that there were significant differences in these characteristics. However, because the case villages and control villages were not necessarily comparable, caution is necessary when interpreting the 
results. If case and control individuals are recruited in the same village, then there might be no significant differences in individual and household characteristics. However, a previous case-control study that recruited cases and controls in the same village in Lao PDR also showed that there were significant differences in individual and household characteristics, i.e., not using a bed net, sleeping away from home, and houses closed to a vector breeding site [8].

A cross-sectional study that was conducted in Xepon district by Pongvongsa et al. showed that malaria infections were highly clustered at the household level. Pongvongsa et al. suggested two possible reasons for such household clustering. One is that household members share the same environmental risk factors including the proximity of housing location to breeding sites and housing type. Another is that household members share the same risk behaviors such as not using a bed net and working in the forest [30]. The results of the present study partly support their speculation. The present study found that houses with an open space were significantly more common in villages with high incidences than in those with low incidences of malaria, suggesting the importance of housing conditions. The present study also found that some households did not own any LLINs, suggesting that all members of the households are equally not protected by nets. The present study, however, did not find that risk behaviors were highly clustered at the household level; individuals who practiced risk behaviors did not concentrate on particular households.

There are four limitations in the present study. Firstly, the case villages were not matched with the control villages on the basis of village-level characteristics. Therefore, the risk of malaria infection could be different between the two. The stratum III case villages were more likely to be located far from the health center as compared with the stratum I and II control villages. Thus, the case villages were likely to be at higher risk of malaria infections, as compared to control villages. The ethnicity of the study population was, however, the same between the case and control villages, i.e., Tri and Mangkong. To minimize the impact of the absence of matching, the present study excluded the stratum I and II villages that were located within $7 \mathrm{~km}$ of the health center. Secondly, although people in the study site might have sought malaria treatment from the private sector, the village-level incidence of malaria used in the present study relied solely on records from the public health sector. Thus, the present study could underestimate the village-level incidence. However, the impact of the underestimation would be very low because access to the private health sector was available only in the center of Xepon district, which was located at least $8 \mathrm{~km}$ from the study villages, and as shown in Table 2, more than $90 \%$ of the study households reported treatment seeking from the public sector for past malaria episodes. Thirdly, because in the present study the outcome measurement was not at the individual level but at the village level, the present study was unable to take the village and household clustering into account in the analyses. Therefore, the present study possibly overestimated the significance of the statistical associations. Finally, because small villages in which the total number of households was 14 or fewer were excluded, the results of the present study were not free from selection bias. However, the impact of the selection bias would be small as the total number of households was 15 or larger in the most of the villages of the study district.

Although in the present study the study site was confined to the catchment area of one health center in Xepon district, the findings of the present study can be generalized to a wider area in Savannakhet province. This is because the same ethnic groups such as Tri and Mangkong are predominant in the malaria-prone villages of the province including the study villages of the present study. Additionally, ecological characteristics are similar among the malaria-prone villages of the province.

\section{Conclusion}

There were significant differences in personal behaviors and household environment between the villages with high and those with low incidences of malaria. Villagers in the villages with high incidences of malaria were significantly more likely to work and sleep in the forest at night as compared with those in villages with low incidences. Houses in villages with high incidences were significantly more likely to have open spaces on their surfaces that could facilitate mosquito entry. These differences can partly explain the difference in the incidences of malaria between the villages. The Lao National Malaria Control Program should recommend that villagers use personal protection such as repellent lotions, single LLINs, and long-sleeved clothes when they work and sleep in the forest and to reduce open spaces on house surfaces by installing net screens.

\footnotetext{
Acknowledgements

The authors thank the study participants and heads of the study villages for their cooperation. The authors also thank Dongsavanh Health Center, Xepon District Health Office, and Savannakheth Provincial Health Department for their contributions.
}

\section{Funding}

This study was supported by a JICAVAMED SATREPS project for the "Development of innovative research technique in genetic epidemiology of malaria and other parasitic diseases in the Lao PDR for containing their expanding endemicity" and the Grant for National Center for Global Health and Medicine (28-4). 


\section{Availability of data and materials}

Raw data may be obtained from the corresponding author upon request.

\section{Authors' contributions}

$\mathrm{NI}$ was the principal investigator and drafted the manuscript with the help of DN, MI, and SKA. SKO, SP, JK, BH, and PTB contributed to the conception of the study. TP contributed to data collection. DN contributed to the data analysis. All of the authors have read and approved the final manuscript.

\section{Ethics approval and consent to participate}

This study was approved by the National Ethics Committee for Health Research, Ministry of Health, Lao PDR (No. 028/NECHR, 11/11/2016) and the Ethics Review Committee for Epidemiological Study, University of the Ryukyus, Japan (No. 362). Before starting the survey, surveyors explained to the participants the details of this study, such as its purpose, voluntary participation, information that would be collected, and how to keep and manage the data. Written informed consent was obtained from each respondent.

\section{Consent for publication}

Not applicable

\section{Competing interests}

The authors declare that they have no competing interests.

\section{Publisher's Note}

Springer Nature remains neutral with regard to jurisdictional claims in published maps and institutional affiliations.

\section{Author details}

'Department of Global Health, Graduate School of Health Sciences, University of the Ryukyus, Uehara 207, Nishihara-cho, Okinawa 903-0215, Japan. ${ }^{2}$ SATREPS Project for Parasitic Diseases, Vientiane, Lao People's Democratic Republic. ${ }^{3}$ National Institute of Public Health, Ministry of Health, Ban Kaognot, Samsenthai Road, Sisattanak District, Vientiane, Lao People's Democratic Republic. ${ }^{4}$ Department of Tropical Medicine and Malaria, Research Institute, National Center for Global Health and Medicine, 1-21-1 Toyama, Shinjuku-ku, Tokyo 162-8655, Japan. ${ }^{5}$ Institut Pasteur du Laos, Ministry of Health, Sisattanak District, Vientiane, Lao People's Democratic Republic. ${ }^{6}$ Center of Malariology, Parasitology and Entomology, Ministry of Health, Vientiane, Lao People's Democratic Republic. 'Savannakhet Provincial Health Department, Thahea village, Kaysone-Phomvihan District, Savannakhet, Lao People's Democratic Republic.

Received: 14 August 2017 Accepted: 25 October 2017 Published online: 07 November 2017

\section{References}

1. Lao Statistics Bureau. Vientiane capital: Ministry of Planning and Investment, Lao PDR. 2016. http://www.Isb.gov.la/lsb/en/\#.WfcXzVu0000. Accessed 30 Oct 2017.

2. Midgley S, Bennett J, Samontry X, Stevens P, Mounlamai K, Midgley D, et al. Enhancing livelihoods in Lao PDR through environmental services and planted-timber products. Canberra: Australian Centre for International Agricultural Research; 2012. p. 14-5.

3. Ministry of Health: Lao People's Democratic Republic. National strategy for malaria control and pre-elimination 2011-2015. Lao People's Democratic Republic: Ministry of Health; 2010.

4. Ministry of Health: Lao People's Democratic Republic. National strategy for malaria control and elimination 2016-2030. Lao People's Democratic Republic: Ministry of Health; 2016.

5. Kounnavong S, Gopinath D, Hongvanthong B, Khamkong C, Sichanthongthip O. Malaria elimination in Lao PDR: the challenges associated with population mobility. Infect Dis Poverty. 2017;6:81.

6. Nonaka D, Laimanivong S, Kobayashi J, Chindavonsa K, Kano S, Vanisaveth $V$, et al. Is staying overnight in a farming hut a risk factor for malaria infection in a setting with insecticide-treated bed nets in rural Laos? Malar J. 2010;9:372.

7. Khaminsou N, Kritpetcharat O, Daduang J, Kritpetcharat P. A survey of malaria infection in endemic areas of Savannakhet province, Lao PDR and comparative diagnostic efficiencies of Giemsa staining, acridine orange staining, and semi-nested multiplex PCR. Parasitol Int. 2008;57:143-9.

8. Vythilinggam I, Sidavong B, Chan ST, Phonemixay T, Vanisaveth V, Sisoulad $P$, et al. Epidemiology of malaria in Attapeu province, Lao PDR in relation to entomological parameters. Trans $\mathrm{R}$ Soc Trop Med Hyg. 2005;99:833-9.

9. Somboon P, Aramrattana A, Lines J, Webber R. Entomological and epidemiological investigations of malaria transmission in relation to population movements in forest areas of north-west Thailand. Southeast Asian J Trop Med Public Health. 1998;29:3-9.

10. Gunawardena DM, Wickremasinghe AR, Muthuwatta L, Weerasingha S, Rajakaruna J, Senanayaka T, et al. Malaria risk factors in an endemic region of Sri Lanka, and the impact and cost implications of risk factor-based interventions. Am J Trop Med Hyg. 1998;58:533-42.

11. Hiscox A, Khammanithong P, Kaul S, Sananikhom P, Luthi R, Hill N, et al. Risk factors for mosquito house entry in the Lao PDR. PLoS One. 2013;8:5.

12. Abe T, Honda S, Nakazawa S, Tuong TD, Thieu NQ, Hungle X, et al. Risk factors for malaria infection among ethnic minorities in Binh Phuoc, Vietnam. Southeast Asian J Trop Med Public Health. 2009:40:18-29.

13. Erhart A, Thang ND, Ky PV, Tinh $\Pi$, Overmeir CV, Speybroeck N, et al. Epidemiology of forest malaria in central Vietnam: a large scale crosssectional survey. Malar J. 2005;4:58.

14. Chaveepojnkamjorn W, Pichainarong N. Behavioral factors and malaria infection among the migrant population, Chiang Rai province. J Med Assoc Thail. 2005;88:1293-301.

15. Nonaka D, Pongvongsa T, Nishimoto F, Nansounthavong P, Sato $Y$, Jiang $H$, et al. Households with insufficient bednets in a village with sufficient bednets: evaluation of household bednet coverage using bednet distribution index in Xepon district. Lao PDR Trop Med Health. 2015:43:95-100.

16. Vyas S, Kumaranayake L. Constructing socio-economic status indices: how to use principal components analysis. Health Policy Plan. 2006;21: 459-68.

17. Katz HM. Multivariable analysis: a practical guide for clinicians and public health researchers. 3rd ed. Cambridge: Cambridge University Press; 2011.

18. Phommasone K, Adhikari B, Henriques G, Pongvongsa T, Phongmany P, von Seidlein $L$, et al. Asymptomatic plasmodium infections in 18 villages of southern Savannakhet province, Lao PDR (Laos). Malar J. 2016;15:296.

19. Pongvongsa T, Ha H, Thanh L, Marchand RP, Nonaka D, Tojo B, et al. Joint malaria surveys lead towards improved cross-border cooperation between Savannakhet province, Laos and Quang Tri province. Vietnam Malar J. 2012;11:262.

20. Kobayashi J, Phompida S, Toma T, Looareensuwan S, Toma H, Miyagi I. The effectiveness of impregnated bed net in malaria control in Laos. Acta Trop. 2004;89:299-30.

21. Pichainarong N, Chaveepojnkamjorn W. Malaria infection and life-style factors among hilltribes along the Thai-Myanmar border area, northern Thailand. Southeast Asian J Trop Med Public Health. 2004;35:834-9.

22. Lindsay SW, Emerson PM, Charlwood JD. Reducing malaria by mosquitoproofing houses. Trends Parasitol. 2002;18:510-4.

23. Kirby MJ, Ameh D, Bottomley C, Green C, Jawara M, Milligan PJ, et al. Effect of two different house screening interventions on exposure to malaria vectors and on anaemia in children in the Gambia: a randomised controlled trial. Lancet. 2013;374:998-1009.

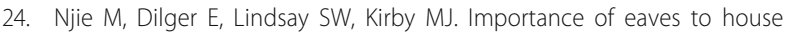
entry by anopheline, but not culicine, mosquitoes. J Med Entomol. 2009:46:505-10

25. Ghebreyesus TA, Haile M, Witten KH, Getachew A, Yohannes M, Lindsay SW, et al. Household risk factors for malaria among children in the Ethiopian highlands. Trans R Soc Trop Med Hyg. 2000;94:17-21.

26. Tusting LS, Ippolito M, Willey B, Kleinschmidt I, Dorsey G, Gosling RD, et al. The evidence for improving housing to reduce malaria: a systematic review and meta-analysis. Malar J. 2015;14:209.

27. Kirby MJ, Bah P, Jones CO, Kelly AH, Jasseh M, Lindsay SW. Social acceptability and durability of two different house screening interventions against exposure to malaria vectors, plasmodium falciparum infection, and anemia in children in the Gambia, West Africa. Am J Trop Med Hyg. 2010;83:965-72. 
28. Tusting LS, Rek J, Arinaitwe E, Staedke SG, Kamya MR, Cano J, et al. Why is malaria associated with poverty? Find Cohort Study in Rural Uganda Malar J. 2016;5:78.

29. Snow RW, Peshu N, Forster D, Bomu G, Mitsanze E, Ngumbao E, et al. Environmental and entomological risk factors for the development of clinical malaria among children on the Kenya coast. Trans R Soc Trop Med Hyg. 1998;92:381-5.

30. Pongvongsa T, Nonaka D, Iwagami M, Nakatsu M, Phongmany P, Nishimoto F, et al. Household clustering of asymptomatic malaria infections in Xepon district, Savannakhet province. Lao PDR Malar J. 2016;15:508.

Submit your next manuscript to BioMed Central and we will help you at every step:

- We accept pre-submission inquiries

- Our selector tool helps you to find the most relevant journal

- We provide round the clock customer support

- Convenient online submission

- Thorough peer review

- Inclusion in PubMed and all major indexing services

- Maximum visibility for your research

Submit your manuscript at www.biomedcentral.com/submit
C) Biomed Central 